34-14941 to genes). For each module, we defined a quantitative measure of module membership as the correlation of the module eigengene with the gene expression profile. Modules were phenotypically stratified based on weight, age, mortality, and organ dysfunction. A heatmap plot was generated of the adjacencies in the eigengene network (figure 3). Genes from the significant modules showing high module membership were filtered and selected (p.MM s 0.05 ). Number of Genes related to trait included 740 (mortality) 2151 (weight) and 1616 (age). Weight was correlated high ( $\mathrm{r} 0.53$ and $\mathrm{p}$ value 0.003 ) with the purple module (MEpurple) to weight (figure 4).

Conclusions Our analysis shows that using the time series transcriptome in MenS, novel associations can be identified that could influence future treatment options for improved outcomes.

\section{EXPANDED CARRIER SCREENING: PRIMARY PREVENTION OF RECESSIVE MONOGENIC DISEASES EVALUATED USING 1,909 CHINESE GENOME AND EXOME SEQUENCING DATA}

Fong Ting Jeffrey Chau, Ho Chung Mullin Yu, Sze-Man Clara Tang, Kit San Yeung, Hon-Yin Brian Chung. Hong Kong

\subsection{6/bmjpo-2021-RCPCH.93}

Background Expanded carrier screening (ECS) is a genetic test that investigates the genetic composition of a couple and determines whether their offspring has an elevated risk of inherited disorders. Comparisons between commercially available ECS has, however, only shown small overlaps in common genes offered for screening.

Objectives Compiled with the inadequate information surrounding carrier frequencies in the Chinese population, secondary usage of next generation sequencing could be used in the optimization of ECS panels surrounding clinical utility, public health benefits, and reducing unnecessary socio-psychological stress.

Methods In this study, a total of 1543 Southern Chinese and 366 Northern Chinese genome and exome sequencing were screened for carrier status over 315 genes. The gene list curated for this study was compiled from three ECS panels offered by frequently used commercial companies and literature reporting high carrier frequencies for treatable inherited disorder in South East Asia population genomics.

Results 180 unique disease-causing variants were identified and $47.8 \% \quad(\mathrm{n}=738)$ of Southern Chinese individuals screened in this study harboured at least 1 disease-causing variant. CNV calling determined 4 unique pathogenic or likely pathogenic copy number variants. A total of 285 unique carrier variants were classified as pathogenic or likely pathogenic. Results have also identified 12 genes with a carrier frequency over $1 \%$ including GJB2, HBA1/HBA2, SMN1, SLC22A5, SLC25A13, ATP7B, SLC26A4, GALC, POLG, USH2A, and HBB.

Conclusions This study shows that secondary analysis of NGS data can illustrate the carrier frequencies in the Southern Chinese population. Through the comparison of different commercially available ECS panels, we identified potential for improvement in the optimization of commercially available ECS panels for the future of precision medicine.

\section{DISCHARGE MANAGEMENT OF CHILDREN UNDER 5 WITH SUSPECTED ASTHMA IN A UK HOSPITAL}

Charlotte Ford, Godfrey Nyamugunduru, Sarah Woolf. UK

\subsection{6/bmjpo-2021-RCPCH.94}

Background The British Thoracic Society (BTS) recommends that children under 5 years of age who are admitted with suspected asthma should be discharged as per the BTS Asthma Discharge Care Bundle since this improves patient outcomes ${ }^{1}$. Many children with suspected asthma are prescribed inhaled corticosteroids (ICS) to reduce airway inflammation ${ }^{2}$. We suspected substandard discharge care of children under 5 hence decided to audit our practice.

Objectives

1. To assess compliance with the BTS asthma discharge bundle, for children under 5 admitted with suspected asthma, who were discharged on inhaled corticosteroids.

2. To improve and standardise the discharge planning of this group of children.

Methods Children with a discharge diagnosis of viral induced wheeze (VIW) or asthma, between 01/12/2018 and $31 / 11 / 2019$, were provided by the hospital information department. Patient notes on iSOFT electronic patient record system were reviewed and those discharged on ICS were identified. From these, every other case in 'date of admission order' was selected and audited against the BTS bundle. This retrospective audit was approved by the trust.

Results Out of a total of 447 children discharged with VIW or asthma under the age of five, 123 patients were discharged on ICS and of these 62 were randomly selected for audit.

Only 9/62 (15\%) patient records were 100\% compliant with the BTS bundle. 22/62 (35\%) were less than 50\% compliant. 17/62 (27\%) did not have any follow up (neither hospital nor community).

\begin{tabular}{ll} 
Abstract 172 Table 1 & \\
\hline BTS discharge care standards & Compliance ( $\mathbf{n}=62)$ \\
\hline Community follow up within two days & $13(21 \%)$ \\
Importance of adherence to preventer medication discussed. & $19(31 \%)$ \\
Triggers considered- including smoke exposure. & $24(39 \%)$ \\
Specialist care follow up in a month. & $27(44 \%)$ \\
Inhaler technique assessed. & $32(52 \%)$ \\
Consultant appointment in 2-3 months. & $41(66 \%)$ \\
Inhaler use instruction provided. & $50(81 \%)$ \\
Medications reviewed prior to discharge. & $55(89 \%)$ \\
Written action plan provided. & $59(95 \%)$ \\
\hline
\end{tabular}

Conclusions The BTS discharge standards for children under 5 years with suspected asthma were not followed in the majority of children. The lack of follow up of many children who were discharged on ICS is particularly concerning, potentially sentencing them to long term ICS and consequent risk of side effects.

We recommend that shortfalls identified by this audit be addressed followed by re-audit after a year. 